\title{
Simple, Optimal and Efficient Auctions
}

\author{
Constantinos Daskalakis $^{1 \star}$ and George Pierrakos ${ }^{2 \star \star}$ \\ 1 MIT, EECS, CSAIL, costis@csail.mit.edu, \\ 2 UC Berkeley, EECS, georgios@cs.berkeley.edu
}

\begin{abstract}
We study the extent to which simple auctions can simultaneously achieve good revenue and efficiency guarantees in single-item settings. Motivated by the optimality of the second price auction with monopoly reserves when the bidders' values are drawn i.i.d. from regular distributions [12], and its approximate optimality when they are drawn from independent regular distributions [11], we focus our attention to the second price auction with general (not necessarily monopoly) reserve prices, arguably one of the simplest and most intuitive auction formats. As our main result, we show that for a carefully chosen set of reserve prices this auction guarantees at least $20 \%$ of both the optimal welfare and the optimal revenue, when the bidders' values are distributed according to independent, not necessarily identical, regular distributions. We also prove a similar guarantee, when the values are drawn i.i.d. from a-possibly irregular-distribution.
\end{abstract}

\section{Introduction}

Social welfare and revenue are without doubt the two most important objectives in mechanism design. They are both well-studied, and extremely well-understood when there is a single item for sale. Not only do the Vickrey and Myerson auctions optimize these objectives in isolation, but there also exist (typically randomized) mechanisms that simultaneously optimize for both objectives, in the sense of maximizing revenue subject to a lower bound on social welfare, or vice-versa [13]. Interestingly, when the bidders' values are independently and identically distributed according to some regular distribution, ${ }^{3}$ the Vickrey and Myerson mechanisms behave very much alike: Myerson's auction is just Vickrey's auction with an additional reserve price. Motivated by this astonishing similarity (and the somewhat peculiar format of Myerson's auction in more general settings), Hartline and Roughgarden [11] showed that a Vickrey auction with appropriately chosen reserve prices can approximate the revenue of the optimal auction in more general settings. Inspired by their result, and the fact that

* Supported by a Sloan Foundation Fellowship and NSF Award CCF-0953960 (CAREER) and CCF-1101491.

** Research (partially) supported by NSF grant CC-0964033 and by a Google University Research Award. Part of this work was done while the author was visiting Microsoft Research, New England.

${ }^{3}$ See Sec. 2 for a definition. 
the mechanism of [13] is at least as complicated as Myerson's mechanism and potentially randomized, in this paper we ask the question of whether one can design simple and deterministic mechanisms that achieve approximately-optimal guarantees for both objectives simultaneously.

At first glance it is not obvious why such simple auctions should even exist. Indeed, despite the fact that Vickrey's auction achieves at least half of the optimal revenue, when the values are drawn i.i.d. from regular distributions (see e.g. [9]), this is no longer the case when the values are independent but drawn from different regular distributions. In particular, it is easy to see that the revenue of Vickrey's auction can be arbitrarily far from the optimal revenue: just consider $n-1$ bidders distributed independently and uniformly in $[0,1]$, and a single bidder distributed uniformly in $[h, h+1]$, for some large $h>1$. The situation does not become any better if we resort to the mechanism of [11], i.e. running Vickrey with a different reserve price for every bidder, taken to be Myerson's monopoly reserve price for that bidder. The auction now can be arbitrarily inefficient even for a single bidder whose value is distributed according to a regular distribution: consider the (almost) equal revenue distribution, where the bidder's value is supported on $\{1-\epsilon, 2-\epsilon, \ldots, h-\epsilon\}$, for some $\epsilon \in(0,1)$ and $h>1$, and the probability that it is larger than or equal to $i-\epsilon$ is exactly $1 / i$, for $i=1 \ldots h$. In this paper, we show that by appropriately tweaking the reserve price of each bidder, we can fix this inefficiency:

Main Result (Th. 1 of Sec. 3): In every single-item setting with $n$ bidders whose values are distributed according to independent (possibly non-identical) regular distributions and for every $p \in[0,1]$, there exists a Vickrey auction with (generally non-anonymous) reserve prices that simultaneously achieves a $p$ fraction of the optimal social welfare and a $\left(\frac{1-p}{4}\right)$-fraction of the optimal revenue. In particular, there exists a Vickrey auction with reserve prices that achieves at least a $20 \%$ of the optimal social welfare and revenue.

We can use our techniques to prove a similar approximation guarantee for non-identical distributions satisfying the monotone hazard rate condition (which has already been obtained by [7]), and we also show that a Vickrey auction with an anonymous reserve simultaneously approximates both objectives for general (possibly non-regular) distributions, as long as all values are i.i.d (Th. 3). We summarize our results together with already known welfare and revenue guarantees for various settings in Table 1.

\begin{tabular}{|c||c|c|}
\hline & i.i.d. & independent \\
\hline \hline mhr & $\left(1, \frac{1}{e}\right)$ and $\left(\frac{1}{e}, 1\right)[2]$ & $\left(\frac{1}{e}, \frac{1}{2}\right)[7]$ \\
\hline regular & $\left(1, \frac{1}{2}\right)[9]$ & $\left(\frac{1}{5}, \frac{1}{5}\right)$ and $\left(p, \frac{1-p}{4}\right)$, for all $p \in[0,1]$ [this work] \\
\hline non-regular & $\left(\frac{1}{2}, \frac{1}{2}\right)[$ this work] & $?$ \\
\hline
\end{tabular}

Table 1. $(\alpha, \beta)$ stands for $\alpha$-approximation for welfare and $\beta$-approximation for revenue. Notice that our result for regular distributions gets a handle on the whole Pareto boundary achieved by the Vickrey auction with non-anonymous reserve prices. 
Two questions left open are whether one can extend our results to the setting of $n$ bidders distributed according to independent but not necessarily identical and possibly irregular distributions, and to general single-dimensional settings.

\section{$1.1 \quad$ Related Work}

The work closer in spirit to ours is that of [11], where the authors show that for a variety of single-dimensional settings, second price auctions with carefully chosen reserve prices are approximately revenue-optimal. In particular, when the bidders' values are independently drawn from (possibly different) regular distributions, they show that Vickrey's auction with monopoly reserve prices (see Sec. 2 for a definition) achieves at least half of the optimal revenue. Moreover they show that Vickrey's auction with an anonymous reserve achieves a factor 4 approximation to the optimal revenue.

In an unpublished manuscript [8], the authors study the problem of designing deterministic mechanisms that optimize for both objectives, as a multi-objective optimization problem. They show that, even though exactly optimizing the trade-off curve is an NP-hard problem, there exists a polynomial-time deterministic mechanism that approximates within arbitrary precision any point on the trade-off curve of those two objectives, when there are 2 bidders with arbitrarily correlated values. Their mechanism, despite being deterministic, is far from simple; this work complements theirs by showing that, if one is willing to settle for less than an arbitrarily small approximation factor, simple mechanisms are possible, even when the number of bidders is large. Moreover, the existence of an auction that simultaneously achieves a constant factor approximation to both objectives, characterizes the "knee" of the Pareto curve, a structural result which is of independent interest.

A different type of result relating the two objectives is that of Bulow and Klemperer [4], where it is shown that in a single-item setting the revenue benefits of adding an extra bidder and running the efficiency-maximizing (Vickrey) auction surpass those of running the revenue-maximizing (Myerson) auction without adding the extra bidder, when the bidders' values are i.i.d. according to a regular distribution. In [2] the authors show that for values drawn i.i.d. from a monotone hazard rate distribution, an analogous theorem holds for efficiency: by adding $\Theta(\log n)$ extra bidders and running Myerson's auction, one gets at least the efficiency of Vickrey's auction. Finally, [11] extends Bulow and Klemperer's result to more general single-dimensional settings, as follows: they show that by duplicating all bidders (whose values are drawn independently from not necessarily identical, regular distributions), and then running the VCG auction, one can guarantee at least half of the optimal revenue (while being optimal with respect to welfare). Our result shows that in single-item settings with independent (but not necessarily i.i.d.) bidders, one can simultaneously achieve constant factor approximations to both optimal revenue and welfare without adding any extra bidders via the use of a Vickrey auction with appropriate (non-anonymous) reserve prices. 
There has also been substantial work studying the revenue and welfare guarantees of welfare-optimizing and revenue-optimizing auctions respectively. In [2] the authors show that, for values drawn independently from the same monotone hazard rate distribution, both the welfare and revenue ratios of Vickrey and Myerson's auctions are bounded by 1/e (see the top-left square of Table 1). Similar kinds of revenue and welfare ratios are also studied in [9] for keyword auctions, in [14] for single-item English auctions, and in [1], where the authors present bounds on the efficiency loss of revenue-optimal mechanisms in singleitem settings with i.i.d. bidders of finite support. Moreover, in [9] and [7] the authors present simple auctions that simultaneously achieve constant factor approximations to both objectives in single-item settings where bidders' values are i.i.d. from a regular distribution (see the middle-left square of Table 1), and independently (but not necessarily identically) distributed according to a monotone hazard rate distribution (see the top-right square of Table 1). Some of their results also hold for more general single-dimensional settings, namely when the feasibility constraints form a matroid.

Finally, despite our different motivation, methodologically our paper is somewhat related to [3]: in that paper the goal is to provide a general reduction from the mechanism design problem for many bidders, to that of a single bidder, while preserving the value of a separable objective (such as welfare or revenue) within a constant factor. In Lem. 3 and 5 we establish analogous many-to-one reductions; however, our goal is not only to preserve the approximation factor, but also for the resulting auction to be of a specific simple format, in contrast to the much more generic reduction of [3].

\section{Preliminaries}

Our auction setting is that of a single item for sale and $n$ interested bidders, each with a value $v_{i}$ for the item, which is distributed independently according to some distribution $F_{i}$. The distributions $\left\{F_{i}\right\}_{i}$ are not necessarily identical. For simplicity we assume that all $F_{i}$ 's in this paper are differentiable. So we can define the corresponding probability density functions as follows $f_{i}(x)=F_{i}^{\prime}(x)$.

A single-item auction $\mathcal{A}$ consists of an allocation rule $\mathbf{x}$ and a payment rule $\mathbf{p}$; an allocation rule is a function from bid vectors to $[0,1]^{n}$, encoding the probability by which every bidder receives the item, while a payment rule is a function from bid vectors to $n$-vectors of non-negative payments. We want from our auctions to satisfy the two standard constraints of ex-post incentive compatibility (IC) and individual rationality (IR) [12], so that the terms "bid" and "value" can be used interchangeably. We are interested in the objectives of revenue and welfare, defined as follows:

$$
\operatorname{Rev}[\mathcal{A}]=\mathbb{E}\left[\sum_{i=1}^{n} p_{i}\left(v_{1}, \ldots, v_{n}\right)\right] \text { and } \operatorname{SW}[\mathcal{A}]=\mathbb{E}\left[\sum_{i=1}^{n} v_{i} \cdot x_{i}\left(v_{1}, \ldots, v_{n}\right)\right]
$$

where the above expectations are with respect to value vectors $v=\left(v_{1}, \ldots, v_{n}\right)$ drawn from the product distribution $\times_{i} F_{i}$. For convenience, we sometimes write $\mathcal{R}_{\mathcal{A}}=\sum_{i=1}^{n} p_{i}\left(v_{1}, \ldots, v_{n}\right)$, so that $\operatorname{Rev}[\mathcal{A}]=\mathbb{E}\left[\mathcal{R}_{\mathcal{A}}\right]$. 
We say that an auction $\mathcal{A}$ is an $\alpha$-approximation for welfare (resp. revenue) if $\operatorname{SW}[\mathcal{A}] \geq \alpha \cdot \operatorname{SW}[\mathrm{Vic}](\operatorname{resp} . \operatorname{Rev}[\mathcal{A}] \geq \alpha \cdot \operatorname{Rev}[\mathrm{Mye}]$ ), where Vic denotes the Vickrey auction and Mye denotes Myerson's auction. We say that an auction is an $(\alpha, \beta)$-approximation if it is simultaneously an $\alpha$-approximation for welfare and a $\beta$-approximation for revenue. Also, given an auction $\mathcal{A}$, and a set $B \subseteq\{1, \ldots, n\}$, we may write $\mathcal{A}(B)$ to denote the auction $\mathcal{A}$ run only on the subset $B$ of bidders. When we use this notation it will be clear from context how the "projected" auction operates.

In [12] Myerson introduced the notion of a bidder's virtual valuation function $\phi_{i}$, defined as follows:

$$
\phi_{i}\left(v_{i}\right)=v_{i}-\frac{1-F_{i}\left(v_{i}\right)}{f_{i}\left(v_{i}\right)} .
$$

In terms of this notion, we say a distribution $F_{i}$ is regular if the virtual value function $\phi_{i}$ is non-decreasing, and that it satisfies the monotone hazard rate condition if the ratio $\frac{1-F_{i}(x)}{f_{i}(x)}$ is non-increasing. For distributions that are nonregular, Myerson's ironing technique can be used to get the corresponding ironed virtual valuation function $\hat{\phi}_{i}\left(v_{i}\right)$. The following result is central to Myerson's analysis, and we also use it in the present paper:

Proposition 1. [Myerson's Lemma] For any truthful mechanism (x, p), where all $F_{i}$ are regular distributions, we can express the expected payment of bidder $i$ as follows, where the expectation is over the players' values:

$$
\mathbb{E}\left[p_{i}\left(v_{1}, \ldots, v_{n}\right)\right]=\mathbb{E}\left[\phi_{i}\left(v_{i}\right) \cdot x_{i}\left(v_{1}, \ldots, v_{n}\right)\right] .
$$

We are interested in the following (family) of auction(s):

Definition 1. The Vickrey auction with reserve prices $\mathbf{r}=\left(r_{1}, \ldots, r_{n}\right)$, denoted $\mathrm{Vic}_{\mathbf{r}}$, is the following mechanism:

1. Reject all bidders whose values are $v_{i}<r_{i}$.

2. Allocate the item to the highest valued of the remaining bidders (or to none if no one clears their reserve in Step 1).

3. Charge the winner the maximum of the second highest bidder (among those who were not eliminated in Step 1) and her reserve price.

Tie-break lexicographically if there are multiple highest bidders in Step 2.

Two cases of particular interest are the Vickrey auction with an anonymous reserve, where a common reserve $r$ is used for all bidders, and the Vickrey auction with monopoly reserves, denoted by $\mathrm{Vic}_{\mathbf{m}}$, where $m_{i}=\phi_{i}^{-1}(0)$, the monopoly reserve of bidder $i$.

\section{The regular, independent case}

In this section we focus on the setting of $n$ bidders whose values are distributed according to regular, but not necessarily identical, distributions. We start with a couple of probabilistic lemmas - not requiring regularity- whose easy proofs are deferred to the Appendix. 
Lemma 1. Let $X$ and $Y$ be independent random variables and $g: \mathbb{R} \rightarrow \mathbb{R} a$ (weakly) increasing function. Then, for any constant $c \in \mathbb{R}$,

$$
\operatorname{Pr}[X \geq Y \mid g(X) \geq c] \geq \operatorname{Pr}[X \geq Y \mid g(X) \leq c] .
$$

Lemma 2. Let $X$ and $Y$ be independent random variables and $g: \mathbb{R} \rightarrow \mathbb{R} a$ (weakly) increasing function. Then

$$
\mathbb{E}[g(X)] \leq \mathbb{E}[g(X) \mid X \geq Y]
$$

Our next lemma shows that if we take the Vickrey auction and add a reserve price for each bidder, such that the probability of any single bidder's value exceeding her reserve price is at least $p$, then the resulting welfare is at least a $p$ fraction of Vickrey's (optimal) social welfare $\mathbb{E}\left[\max _{i}\left\{v_{i}\right\}\right]$. The proof of this lemma is relatively straightforward and is deferred to the Appendix as well. In what follows we use $\mathbb{I}_{(\cdot)}$ to denote the indicator function.

Lemma 3. [Many-to-One Reduction-Welfare] Suppose that $X_{1}, \ldots, X_{n}$ are independent, non-negative random variables (possibly non-identically distributed), $t_{1}, \ldots, t_{n}$ are (possibly different) thresholds, and $p \in[0,1]$. If it holds that $\operatorname{Pr}\left[X_{i} \geq t_{i}\right] \geq p$, for all $i=1 \ldots n$, then:

$$
\mathbb{E}\left[\max _{i}\left\{X_{i} \cdot \mathbb{I}_{\left(X_{i} \geq t_{i}\right)}\right\}\right] \geq p \cdot \mathbb{E}\left[\max _{i}\left\{X_{i}\right\}\right] .
$$

Lemma 3 immediately implies the following corollary, already known from [7].

Corollary 1. [mhr, independent] In every single-item setting with $n$ bidders whose values are distributed according to independent (possibly non-identical) distributions that satisfy the monotone hazard rate condition, the Vickrey auction with monopoly reserves is a $(1 / e, 1 / 2)$-approximation.

Proof. It is known from [11] that, if $\mathbf{m}$ is the vector of monopoly reserve prices, then $\mathrm{Vic}_{\mathbf{m}}$ (the Vickrey auction with monopoly reserves) is a $1 / 2$-approximation to the optimal revenue. The welfare guarantee follows from Lem. 3 and the following fact from [2]: if $v$ is drawn from a monotone hazard rate distribution, then $\operatorname{Pr}\left[v \geq \phi^{-1}(0)\right] \geq 1 / e$.

Unfortunately, as discussed in Sec. 1, the Vickrey auction with monopoly reserve prices may be arbitrarily inefficient when we allow for regular distributions; in particular we cannot employ Lem. 3 directly as the probability of any single bidder being above her monopoly reserve may be arbitrarily small. To fix this, we recall a lemma for regular distributions from [5]. For a single bidder setting, this lemma guarantees that there is always a reserve price $r$ (which generally needs to be smaller than the monopoly reserve) that achieves a constant factor of the optimal revenue, while at the same time is smaller than the bidder's value with constant probability. 
Lemma 4 ([5]). Let $F$ be a regular distribution, and let $R_{F}(x)=x \cdot F^{-1}(1-x)$, for all $x \in[0,1],{ }^{4}$ be the revenue curve in quantile space. Then, for all $0<\tilde{q} \leq$ $q \leq p<1$,

$$
R_{F}(\tilde{q}) \leq \frac{1}{1-p} R_{F}(q)
$$

If we try to use Lem. 4 to generalize Cor. 1 to regular distributions, we run into an additional difficulty. Indeed, if we lower the bidders' reserve prices to some vector $\mathbf{r} \leq \mathbf{m}$ below their monopoly reserves and run $\mathrm{Vic}_{\mathbf{r}}$, the bidders will start contributing negative virtual values to the expected virtual welfare of the auction (i.e. its expected revenue). So we need to control the absolute value of the overall negative contribution to the expected virtual social welfare. This is not straightforward and is established in the following lemma, which alongside our main result is one of the main contributions of this paper.

Before providing its proof, it is worth noting that the obvious approach of decomposing the auction's virtual welfare into every bidder's contribution, using the law of total expectation, and then comparing each bidder's contribution under different reserve prices poses technical challenges. In particular, the terms of the decomposition cannot be directly compared as each of these terms depends on the probabilistic experiment that determines the winner of the auction, and this experiment depends on the reserves in ways that makes it hard to find a useful coupling that enables term-by-term comparisons. Our technique tries to disentangle the contribution of each bidder to the virtual welfare of the auction from the competition among the bidders, enabling us to first relate the revenue of $\mathrm{Vic}_{\mathbf{r}}$ with the revenue of a hybrid auction, instead of $\mathrm{Vic}_{\mathbf{m}}$ (for which we have good revenue guarantees from [11]). Our hybrid auction uses the tweaked reserves $\mathbf{r}$ to truncate the bidders' values, but only gives the item to the winner of $\mathrm{Vic}_{\mathbf{r}}$ if the winner also meets her monopoly reserve. Next we relate the revenue of our hybrid auction to $\mathrm{Vic}_{\mathbf{m}}$. This is quite more challenging and involves a calculation that matches events where the hybrid auction makes no sale while $\mathrm{Vic}_{\mathbf{m}}$ makes a sale to events where both auctions make a sale, establishing a factor 2 approximation. We expect our technique to find broader use in mechanism design.

Lemma 5. [Many-to-One Reduction-Revenue] Consider a single-item setting with $n$ bidders whose values are distributed according to independent (possibly non-identical) regular distributions. Let also $\mathbf{r}=\left(r_{1}, \ldots, r_{n}\right)$ be a vector of reserve prices such that, for all $i \in\{1, \ldots, n\}, r_{i} \leq \phi_{i}^{-1}(0)$ (i.e. $r_{i}$ is no larger than the monopoly reserve for bidder i) and $\operatorname{Rev}\left[\operatorname{Vic}_{r_{i}}(\{i\})\right] \geq$ $(1-p) \cdot \operatorname{Rev}[\operatorname{Mye}(\{i\})]$, for some $p \in(0,1)$. (That is, if bidder $i$ were considered in isolation then the Vickrey auction with reserve price $r_{i}$ would achieve a $(1-p)$ fraction of the optimal revenue.) Then it holds that $\operatorname{Rev}\left[\mathrm{Vic}_{\mathbf{r}}\right] \geq \frac{1-p}{4} \cdot \operatorname{Rev}[\mathrm{Mye}]$.

\footnotetext{
${ }^{4}$ See the discussion in [5] for why $F^{-1}$ is a well-defined function for a differentiable regular distribution.
} 
Proof. Let $\mathcal{E}_{i}$ denote the event that $i$ is the winner of the Vikrey auction with reserves r , i.e. $i=\arg \max _{j}\left\{v_{j} \cdot \mathbb{I}_{\left(v_{j} \geq r_{j}\right)}\right\}^{5}$ and $v_{i} \geq r_{i}$. Using Prop. 1 we can write $\operatorname{Rev}\left[\mathrm{Vic}_{\mathbf{r}}\right]$ in terms of the bidders' virtual values as follows:

$$
\begin{gathered}
\operatorname{Rev}\left[\mathrm{Vic}_{\mathbf{r}}\right]=\sum_{i=1}^{n} \mathbb{E}\left[\phi_{i}\left(v_{i}\right) \mid \mathcal{E}_{i}, \phi_{i}\left(v_{i}\right) \in\left[\phi_{i}\left(r_{i}\right), 0\right]\right] \operatorname{Pr}\left[\mathcal{E}_{i}, \phi_{i}\left(v_{i}\right) \in\left[\phi_{i}\left(r_{i}\right), 0\right]\right] \\
+\mathbb{E}\left[\phi_{i}\left(v_{i}\right) \mid \mathcal{E}_{i}, \phi_{i}\left(v_{i}\right) \geq 0\right] \operatorname{Pr}\left[\mathcal{E}_{i}, \phi_{i}\left(v_{i}\right) \geq 0\right]
\end{gathered}
$$

In the course of the proof, we use the following inequalities:

$$
\begin{aligned}
\mathbb{E}\left[\phi_{i}\left(v_{i}\right) \mid \phi_{i}\left(v_{i}\right) \in\left[\phi_{i}\left(r_{i}\right), 0\right]\right] \leq \mathbb{E}\left[\phi_{i}\left(v_{i}\right) \mid \mathcal{E}_{i}, \phi_{i}\left(v_{i}\right) \in\left[\phi_{i}\left(r_{i}\right), 0\right]\right](\leq 0) \\
(0 \leq) \mathbb{E}\left[\phi_{i}\left(v_{i}\right) \mid \phi_{i}\left(v_{i}\right) \geq 0\right] \leq \mathbb{E}\left[\phi_{i}\left(v_{i}\right) \mid \mathcal{E}_{i}, \phi_{i}\left(v_{i}\right) \geq 0\right] \\
\left|\mathbb{E}\left[\phi_{i}\left(v_{i}\right) \mid \phi_{i}\left(v_{i}\right) \in\left[\phi_{i}\left(r_{i}\right), 0\right]\right]\right| \cdot \operatorname{Pr}\left[\phi_{i}\left(v_{i}\right) \in\left[\phi_{i}\left(r_{i}\right), 0\right]\right] \leq \\
p \cdot \mathbb{E}\left[\phi_{i}\left(v_{i}\right) \mid \phi_{i}\left(v_{i}\right) \geq 0\right] \cdot \operatorname{Pr}\left[\phi_{i}\left(v_{i}\right) \geq 0\right]
\end{aligned}
$$

Inequalities (2) and (3) follow from Lem. 2 when $g$ is $\phi_{i}$ and $Y=\max _{j \neq i}\left\{v_{j}\right.$. $\left.\mathbb{I}_{v_{j} \geq r_{j}}\right\}$. Inequality (4) involves a single bidder, and follows immediately from our assumption $\operatorname{Rev}\left[\operatorname{Vic}_{r_{i}}(\{i\})\right] \geq(1-p) \cdot \operatorname{Rev}[\operatorname{Mye}(\{i\})]$ and noting that

$$
\begin{aligned}
\operatorname{Rev}\left[\operatorname{Vic}_{r_{i}}(\{i\})\right] & =\mathbb{E}\left[\phi_{i}\left(v_{i}\right) \mid \phi_{i}\left(v_{i}\right) \in\left[\phi_{i}\left(r_{i}\right), 0\right]\right] \cdot \operatorname{Pr}\left[\phi_{i}\left(v_{i}\right) \in\left[\phi_{i}\left(r_{i}\right), 0\right]\right] \\
& +\mathbb{E}\left[\phi_{i}\left(v_{i}\right) \mid \phi_{i}\left(v_{i}\right) \geq 0\right] \cdot \operatorname{Pr}\left[\phi_{i}\left(v_{i}\right) \geq 0\right] \\
\operatorname{Rev}[\operatorname{Mye}(\{i\})] & =\mathbb{E}\left[\phi_{i}\left(v_{i}\right) \mid \phi_{i}\left(v_{i}\right) \geq 0\right] \cdot \operatorname{Pr}\left[\phi_{i}\left(v_{i}\right) \geq 0\right]
\end{aligned}
$$

Using (2), (3) and (4), we can bound the terms of the negative contribution to the expected revenue (1) as follows:

$$
\begin{aligned}
& \left|\mathbb{E}\left[\phi_{i}\left(v_{i}\right) \mid \mathcal{E}_{i}, \phi_{i}\left(v_{i}\right) \in\left[\phi_{i}\left(r_{i}\right), 0\right]\right]\right| \cdot \operatorname{Pr}\left[\mathcal{E}_{i}, \phi_{i}\left(v_{i}\right) \in\left[\phi_{i}\left(r_{i}\right), 0\right]\right] \\
\leq & \overbrace{}^{\mathbb{E}\left[\phi_{i}\left(v_{i}\right) \mid \phi_{i}\left(v_{i}\right) \in\left[\phi_{i}\left(r_{i}\right), 0\right]\right] \mid \operatorname{Pr}\left[\phi_{i}\left(v_{i}\right) \in\left[\phi_{i}\left(r_{i}\right), 0\right]\right]} \underbrace{\operatorname{Pr}\left[\mathcal{E}_{i} \mid \phi_{i}\left(v_{i}\right) \in\left[\phi_{i}\left(r_{i}\right), 0\right]\right]} \\
\leq & \overbrace{p \cdot \mathbb{E}\left[\phi_{i}\left(v_{i}\right) \mid \phi_{i}\left(v_{i}\right) \geq 0\right] \cdot \operatorname{Pr}\left[\phi_{i}\left(v_{i}\right) \geq 0\right]} \cdot \overbrace{\operatorname{Pr}\left[\mathcal{E}_{i} \mid \phi_{i}\left(v_{i}\right) \geq 0\right]}^{\mathbb{E}\left[\phi_{i}\left(v_{i}\right) \mid \mathcal{E}_{i}, \phi_{i}\left(v_{i}\right) \geq 0\right] \cdot \operatorname{Pr}\left[\mathcal{E}_{i}, \phi_{i}\left(v_{i}\right) \geq 0\right]}
\end{aligned}
$$

where for the first inequality we used (2) (and the fact that both sides of the inequality are non-positive), for the second inequality we used (4) and Lem. 1 taking $g$ equal to $\phi_{i}, X=v_{i}$ (conditioned on $X \geq r_{i}$ ), $Y=\max _{j \neq i}\left\{v_{j} \cdot \mathbb{I}_{v_{j} \geq r_{j}}\right\}$ and $c=0$, and in the third inequality we used (3). We can now bound the revenue as follows:

$$
\operatorname{Rev}\left[\operatorname{Vic}_{\mathbf{r}}\right] \geq(1-p) \cdot \sum_{i=1}^{n} \mathbb{E}\left[\phi_{i}\left(v_{i}\right) \mid \mathcal{E}_{i}, \phi_{i}\left(v_{i}\right) \geq 0\right] \cdot \operatorname{Pr}\left[\mathcal{E}_{i}, \phi_{i}\left(v_{i}\right) \geq 0\right] .
$$

\footnotetext{
5 Throughout the proof we assume that all maximizations have a unique maximizer. This is ok, since we consider continuous distributions so this happens with prob. 1 .
} 
To continue, we observe that the summation on the right-hand-side of (5) can be interpreted as the revenue of the following hybrid auction, $\mathcal{H}$, which lies between $\mathrm{Vic}_{\mathbf{r}}$ and $\mathrm{Vic}_{\mathbf{m}}: \mathcal{H}$ truncates all bidders at their respective reserve prices $r_{i}$; among the surviving bidders it identifies the larger bidder $i^{*}$ as a potential winner, but only allocates the item to $i^{*}$ if she clears her monopoly reserve $m_{i^{*}}$; if this happens, $i^{*}$ pays the maximum of her reserve price $m_{i^{*}}$ and $\max _{j \neq i^{*}}\left\{v_{j} \cdot \mathbb{I}_{\left(v_{j} \geq r_{j}\right)}\right\}$. We can clearly lower bound the expected payment of bidder $i$ in the hybrid auction by the following expression:

$$
\int_{x=0}^{m_{i}} \operatorname{Pr}\left[\max _{j \neq i}\left\{v_{j} \cdot \mathbb{I}_{\left(v_{j} \geq r_{j}\right)}\right\}=x\right] \cdot m_{i} \cdot \operatorname{Pr}\left[v_{i} \geq m_{i}\right] d x .
$$

Hence:

$$
\mathbb{E}\left[\mathcal{R}_{\mathcal{H}}\right] \geq \sum_{i=1}^{n} \int_{x=0}^{m_{i}} \operatorname{Pr}\left[\max _{j \neq i}\left\{v_{j} \cdot \mathbb{I}_{\left(v_{j} \geq r_{j}\right)}\right\}=x\right] \cdot m_{i} \cdot \operatorname{Pr}\left[v_{i} \geq m_{i}\right] d x .
$$

Next we compare the revenue of $\mathcal{H}$ to that of the Vickrey auction with monopoly reserves $\mathrm{Vic}_{\mathbf{m}}$. Our first observation is that whenever (i.e. for any value vector for which) $\mathcal{H}$ sells to some bidder $i$, Vic $\mathbf{c}_{\mathbf{m}}$ also sells to the same bidder $i$; moreover, the payment of bidder $i$ in $\mathcal{H}$ is at least as large as her payment in $\mathrm{Vic}_{\mathbf{m}} \cdot{ }^{6}$ So the contribution of bidder $i$ to the revenue from the event where she gets the item in both auctions is larger in the hybrid auction. This implies that the revenue in the event that both $\mathcal{H}$ and $\mathrm{Vic}_{\mathbf{m}}$ sell the item is larger in $\mathcal{H}$ than $\mathrm{Vic}_{\mathbf{m}}$. Let us call this event the $\operatorname{good}$ event $\mathcal{G}$. We have just argued that

$$
\mathbb{E}\left[\mathcal{R}_{\mathcal{H}} \mid \mathcal{G}\right] \cdot \operatorname{Pr}[\mathcal{G}] \geq \mathbb{E}\left[\mathcal{R}_{\mathrm{Vic}_{\mathbf{m}}} \mid \mathcal{G}\right] \cdot \operatorname{Pr}[\mathcal{G}]
$$

So it suffices to bound the revenue of $\mathrm{Vic}_{\mathbf{m}}$ under the event that $\mathrm{Vic}_{\mathbf{m}}$ sells to some bidder, but the hybrid auction does not sell to any bidder. Let us call this event the bad event, $\mathcal{B}$. We claim that the bad event is contained in the union of the following disjoint events:

$$
B_{i}=\left\{v_{i} \cdot \mathbb{I}_{\left(v_{i} \geq r_{i}\right)}=\max _{j} v_{j} \cdot \mathbb{I}_{\left(v_{j} \geq r_{j}\right)} \text { and } v_{i} \leq m_{i}\right\} \text {, for all } i .
$$

Indeed, if the bad event happens it must be that the winner $j^{*}$ of $\mathrm{Vic}_{\mathbf{m}}$ does not satisfy $v_{j^{*}} \cdot \mathbb{I}_{\left(v_{j^{*}} \geq r_{j^{*}}\right)}=\max _{j}\left\{v_{j} \cdot \mathbb{I}_{\left(v_{j} \geq r_{j}\right)}\right\}$. Suppose instead that $v_{i} \cdot \mathbb{I}_{\left(v_{i} \geq r_{i}\right)}=$ $\max _{j}\left\{v_{j} \cdot \mathbb{I}_{\left(v_{j} \geq r_{j}\right)}\right\}$. For $i$ not to be the winner in the hybrid auction it must be that $v_{i} \leq m_{i}$. Hence $B_{i}$ is satisfied.

\footnotetext{
${ }^{6}$ The reason for this is that $\mathcal{H}$ uses lower reserves to truncate the bidders' values. So if $i$ wins in $\mathcal{H}$ her value is larger than her monopoly reserve as well as all other bidders' values truncated at the reserves $\mathbf{r}$. So her value must also be larger than the other bidders' values truncated at the (higher) monopoly reserves $\mathbf{m}$. By the same token, the second highest truncated value will be higher if truncation happens at $\mathbf{r}$ than if it happens at $\mathbf{m}$.
} 
Now, in event $B_{i}$, the maximum possible revenue that any auction (and hence $\mathrm{Vic}_{\mathbf{m}}$ ) could be making is $\max _{j \neq i} v_{j} \cdot \mathbb{I}_{\left(v_{j} \geq r_{j}\right)}$. Hence, the revenue of $\mathrm{Vic}_{\mathbf{m}}$ from the event $B_{i}$ can be upper bounded as:

$$
\begin{aligned}
\mathbb{E}\left[\mathcal{R}_{\mathrm{Vic}_{\mathbf{m}}} \mid B_{i}\right] & \cdot \operatorname{Pr}\left[B_{i}\right] \leq \int_{x=0}^{m_{i}} \operatorname{Pr}\left[\max _{j \neq i}\left\{v_{j} \cdot \mathbb{I}_{\left(v_{j} \geq r_{j}\right)}\right\}=x\right] \cdot x \cdot \operatorname{Pr}\left[x \leq v_{i} \leq m_{i}\right] d x \\
& \leq \int_{x=0}^{m_{i}} \operatorname{Pr}\left[\max _{j \neq i}\left\{v_{j} \cdot \mathbb{I}_{\left(v_{j} \geq r_{j}\right)}\right\}=x\right] \cdot x \cdot \operatorname{Pr}\left[v_{i} \geq x\right] d x \\
& \leq \int_{x=0}^{m_{i}} \operatorname{Pr}\left[\max _{j \neq i}\left\{v_{j} \cdot \mathbb{I}_{\left(v_{j} \geq r_{j}\right)}\right\}=x\right] \cdot m_{i} \cdot \operatorname{Pr}\left[v_{i} \geq m_{i}\right] d x
\end{aligned}
$$

where the last inequality follows from the definition of the monopoly reserve $m_{i}$.

Hence, the revenue of $\mathrm{Vic}_{\mathbf{m}}$ from the bad event $\mathcal{B}$ can be upper bounded as:

$$
\mathbb{E}\left[\mathcal{R}_{\mathrm{Vic}_{\mathbf{m}}} \mid \mathcal{B}\right] \cdot \operatorname{Pr}[\mathcal{B}] \leq \sum_{i=1}^{n} \mathbb{E}\left[\mathcal{R}_{\mathrm{Vic}_{\mathbf{m}}} \mid B_{i}\right] \cdot \operatorname{Pr}\left[B_{i}\right] \leq \mathbb{E}\left[\mathcal{R}_{\mathcal{H}}\right],
$$

where for the first inequality we used that $\mathcal{B} \subseteq \cup_{i} B_{i}$, and for the second inequality we combined (8) and (6). Combining (9) and (7) we obtain:

$$
\operatorname{Rev}[\mathcal{H}] \geq \frac{1}{2} \cdot \operatorname{Rev}\left[\mathrm{Vic}_{\mathbf{m}}\right] .
$$

The lemma follows by combining (5), (10) and noticing that the revenue of $\mathrm{Vic}_{\mathbf{m}}$ is known by [11] to be a $1 / 2$-approximation to the optimal revenue, i.e. $\operatorname{Rev}\left[\mathrm{Vic}_{\mathbf{m}}\right] \geq \frac{1}{2} \cdot \operatorname{Rev}[\mathrm{Mye}]$.

We are now ready to prove our main theorem:

Theorem 1 (Main). For every single-item setting with $n$ bidders whose values are distributed according to independent (possibly non-identical) regular distributions, and any $p \in[0,1]$, there is a vector of reserve prices $\mathbf{r}=\left(r_{1}, \ldots, r_{n}\right)$ such that $\mathrm{Vic}_{\mathbf{r}}$ is a $(p,(1-p) / 4)$-approximation.

Proof. We argue that, for all $i$, there exists a price $r_{i}$ such that the following are satisfied:

$$
\begin{gathered}
\operatorname{Pr}\left[v_{i} \geq r_{i}\right] \geq p ; \text { and } \\
\operatorname{Rev}\left[\operatorname{Vic}_{r_{i}}(\{i\})\right] \geq(1-p) \cdot \operatorname{Rev}[\operatorname{Mye}(\{i\})] .
\end{gathered}
$$

Indeed, we distinguish two cases. If $1-F\left(\phi_{i}^{-1}(0)\right) \geq p$, we take $r_{i}=\phi_{i}^{-1}(0)$ and the above are satisfied automatically. Otherwise, the existence of a reserve with the above properties is implied by Lem. 4 . Given reserves $r_{1}, \ldots, r_{n}$ as above, the theorem follows immediately from Lem. 3 and 5 .

Picking $p=1 / 5$ we obtain a $(1 / 5,1 / 5)$-approximate mechanism for regular distributions.

Corollary 2. [regular, independent] For every single-item setting with $n$ bidders whose values are distributed according to independent (possibly nonidentical) regular distributions, there exist reserve prices $\mathbf{r}$ such that $\mathrm{Vic}_{\mathbf{r}}$ achieves a (1/5,1/5)-approximation. 


\section{The non-regular, i.i.d. case}

In this section we show that the Vickrey auction with an anonymous reserve price achieves a constant factor approximation to both objectives for general distributions, when the bidders' values are distributed independently but identically. We will follow the approach of [6], which makes use of prophet inequalities [15] to show that this auction achieves a 1/2-approximation to the optimal revenue.

We first describe prophet inequalities. Imagine a gambler facing a series of $n$ games in a casino, one on each of $n$ days. Game $i$ has a prize associated with it, whose value is distributed according to some distribution $F_{i}$. The distributions of the prize values are known to the gambler in advance, but their exact realization is not known in advance, and neither is the order of the games. On day $i$ a game is chosen by an adversary trying to minimize the gambler's profit and its prize value is drawn from the corresponding distribution; the gambler needs to decide whether to pick the prize and leave the casino, or ignore it and keep playing. Clearly the gambler's optimal strategy can be computed using backwards induction; on the other hand, there exists a simple threshold strategy that guarantees the gambler at least half of the expected value of the maximum prize. A threshold strategy is a single value $t$, such that the gambler accepts the first prize $i$ with $v_{i} \geq t$; the proof of the following theorem can be found in $[15,10]$.

Theorem 2. There exists a threshold $t$ such that, independently of the order the games are played, the expected prize of the gambler is at least half of the expected value of the maximum prize, and the probability that the gambler receives a prize is exactly 1/2.

In [6] they leverage this theorem to show that the Vickrey auction with an anonymous reserve price achieves at least half of the optimal revenue. We can easily extend this to show a guarantee for both social welfare and revenue.

Theorem 3. In every single-item setting with $n$ bidders whose values are drawn independently from the same (possibly non-regular) distribution, a Vickrey auction with an anonymous reserve price achieves a $1 / 2$-approximation to both optimal revenue and welfare.

Proof. For the sake of completeness we first sketch the proof for revenue. (For full details we refer the reader to [10].) Observe that the problem a revenueoptimizing auctioneer faces is similar to the gambler's problem described above, if prizes are taken to be the bidders' ironed virtual values (assuming that the gambler's strategy treats all values in every flat region of the ironed virtual valuation functions the same). Indeed, let $t$ be the threshold that is guaranteed by Th. 2 , and pick the reserve price to be $p=\hat{\phi}^{-1}(t)$, where $\hat{\phi}$ denotes the ironed virtual valuation of the bidders. If there are multiple $p$ 's mapped to $t$ by $\hat{\phi}$ pick the smallest such $p$. Given this tie-breaking, observe that the Vickrey auction with reserve price $p$ treats all flat regions in the ironed virtual valuation function the same; hence its revenue is equal to the expected ironed virtual value of the winner (prize picked), which by Th. 2 is at least $1 / 2$ of the optimal expected 
ironed virtual surplus (expected maximum prize). Since the latter is an upper bound to the optimal revenue, the revenue of the Vickrey auction with reserve $p$ is a $1 / 2$-approximation to the optimal revenue. Moreover, Th. 2 guarantees that a prize will be picked with probability at least $1 / 2$, i.e.

$$
\operatorname{Pr}\left[\max _{i}\left\{v_{i}\right\} \geq p\right] \geq 1 / 2 \geq \operatorname{Pr}\left[\max _{i}\left\{v_{i}\right\} \leq p\right] .
$$

Note that the way we defined our tie-breaking rule is important for this to hold. Next we show that this auction achieves at least half of the optimal social welfare:

$$
\begin{aligned}
\mathbb{E}\left[\max _{i}\left\{v_{i}\right\}\right] & =\int_{0}^{p} x \cdot \operatorname{Pr}\left[\max _{i}\left\{v_{i}\right\}=x\right] d x+\int_{p}^{\infty} x \cdot \operatorname{Pr}\left[\max _{i}\left\{v_{i}\right\}=x\right] d x \\
& \leq p \cdot \int_{0}^{p} \operatorname{Pr}\left[\max _{i}\left\{v_{i}\right\}=x\right] d x+\int_{p}^{\infty} x \cdot \operatorname{Pr}\left[\max _{i}\left\{v_{i}\right\}=x\right] d x \\
& \stackrel{(11)}{\leq} p \cdot \int_{p}^{\infty} \operatorname{Pr}\left[\max _{i}\left\{v_{i}\right\}=x\right] d x+\int_{p}^{\infty} x \cdot \operatorname{Pr}\left[\max _{i}\left\{v_{i}\right\}=x\right] d x \\
& \leq \int_{p}^{\infty} x \cdot \operatorname{Pr}\left[\max _{i}\left\{v_{i}\right\}=x\right] d x+\int_{p}^{\infty} x \cdot \operatorname{Pr}\left[\max _{i}\left\{v_{i}\right\}=x\right] d x \\
& =2 \cdot \mathbb{E}\left[\max _{i}\left\{v_{i} \cdot \mathbb{I}_{v_{i} \geq p}\right\}\right]
\end{aligned}
$$

\section{References}

1. Vineet Abhishek and Bruce E. Hajek. Efficiency loss in revenue optimal auctions. In $C D C$, pages 1082-1087, 2010.

2. Gagan Aggarwal, Gagan Goel, and Aranyak Mehta. Efficiency of (revenue-)optimal mechanisms. In ACM Conference on Electronic Commerce, pages 235-242, 2009.

3. Saeed Alaei. Bayesian combinatorial auctions: Expanding single buyer mechanisms to many buyers. FOCS, 2011.

4. Jeremy Bulow and Paul Klemperer. Auctions versus negotiations. American Economic Review, 86(1):180-94, March 1996.

5. Yang Cai and Constantinos Daskalakis. Extreme-value theorems for optimal multidimensional pricing. FOCS, 2011.

6. Shuchi Chawla, Jason D. Hartline, David L. Malec, and Balasubramanian Sivan. Multi-parameter mechanism design and sequential posted pricing. In $S T O C$, pages 311-320, 2010.

7. Peerapong Dhangwatnotai, Tim Roughgarden, and Qiqi Yan. Revenue maximization with a single sample. In ACM Conference on Electronic Commerce, pages 129-138, 2010.

8. Ilias Diakonikolas, Christos Papadimitriou, George Pierrakos, and Yaron Singer. Complexity of efficiency-revenue trade-offs in bayesian auctions.

9. Shaddin Dughmi, Tim Roughgarden, and Mukund Sundararajan. Revenue submodularity. In ACM Conference on Electronic Commerce, pages 243-252, 2009.

10. Jason Hartline. Lectures on approximation in mechanism design. Lecture notes, Northwestern University, 2010. 
11. Jason D. Hartline and Tim Roughgarden. Simple versus optimal mechanisms. In ACM Conference on Electronic Commerce, pages 225-234, 2009.

12. Roger B. Myerson. Optimal auction design. Mathematics of Operations Research, 6:58-73, 1981.

13. Roger B. Myerson and Mark A. Satterthwaite. Efficient mechanisms for bilateral trading. Journal of Economic Theory, 29(2):265-281, April 1983.

14. Zvika Neeman. The effectiveness of english auctions. Games and Economic Behavior, 43(2):214-238, May 2003.

15. Ester Samuel-Cahn. Comparison of threshold stop rules and maximum for independent nonnegative random variables. The Annals of Probability, 12(4):pp. 1213-1216, 1984.

\section{A Missing Proofs}

Before stating the proofs, we remind the reader the notion of stochastic dominance of measures.

Definition 2 (Stochastic Dominance). Let $\mathcal{F}_{1}$ and $\mathcal{F}_{2}$ be distributions over $\mathbb{R}$. We say that $\mathcal{F}_{2}$ stochastically dominates $\mathcal{F}_{1}$ iff there exist random variables $X_{1}$ and $X_{2}$ that are marginally distributed according to $\mathcal{F}_{1}$ and $\mathcal{F}_{2}$ respectively, and a coupling of $X_{1}$ and $X_{2}$ such that $X_{1} \leq X_{2}$, with probability 1 .

Proof (of Lemma 1). Fix $c$, let $\mathcal{I}:=\{x \mid g(x)=c\}$ and, without loss of generality, assume that $\operatorname{Pr}[g(x) \leq c] \leq \operatorname{Pr}[g(x) \geq c]$. Let $\mathcal{F}_{1}$ be the distribution of $X$, conditioning on $g(X) \leq c$, and $\mathcal{F}_{2}$ the distribution of $X$, conditioning on $g(X) \geq c$. We claim that $\mathcal{F}_{2}$ stochastically dominates $\mathcal{F}_{1}$. Indeed, let $X_{1}$ be a random variable distributed according to $\mathcal{F}_{1}$ and $X_{2}$ a random variable distributed according to $\mathcal{F}_{2}$. Define any coupling of $X_{1}$ and $X_{2}$ enforcing that whenever $X_{2} \in \mathcal{I}, X_{1}=X_{2}$. This is easy to achieve since, at every point $x \in \mathcal{I}, \mathcal{F}_{1}$ has more probability mass than $\mathcal{F}_{2}$ (using our assumption that $\operatorname{Pr}[g(x) \leq c] \leq \operatorname{Pr}[g(x) \geq c]$.) It is now easy to verify that any such coupling satisfies that $X_{1} \leq X_{2}$, with probability 1 . (For completeness, we note that, if instead we had $\operatorname{Pr}[g(x) \leq c] \geq \operatorname{Pr}[g(x) \geq c]$, we would pick any coupling satisfying that whenever $X_{1} \in \mathcal{I}, X_{2}=X_{1}$.)

Suppose that $X_{1}$ and $X_{2}$ are coupled as above and sample $Y$ independently from $X_{1}$ and $X_{2}$. In the joint distribution $\mathcal{F}$ thus defined, whenever $X_{1} \geq Y$, it must also be that $X_{2} \geq Y$ (since by stochastic domination $\operatorname{Pr}\left[X_{2} \geq X_{1}\right]=$ 1). Hence under $\mathcal{F}: \operatorname{Pr}\left[X_{2} \geq Y\right] \geq \operatorname{Pr}\left[X_{1} \geq Y\right]$. The lemma now follows by simply noticing that the marginal of $\mathcal{F}$ over the pair $\left(X_{1}, Y\right)$ is identical to the distribution of $X$ and $Y$ conditioning on $g(X) \leq c$, and similarly for $\left(X_{2}, Y\right)$.

Proof (of Lemma 2). For any constant $c \in \mathbb{R}$ :

$$
\begin{aligned}
\mathbb{E}[g(X)] & =\mathbb{E}[g(X) \mid X \geq c] \operatorname{Pr}[X \geq c]+\mathbb{E}[g(X) \mid X \leq c] \operatorname{Pr}[X \leq c] \\
& =\mathbb{E}[g(X) \mid X \geq c]+(\mathbb{E}[g(X) \mid X \leq c]-\mathbb{E}[g(X) \mid X \geq c]) \cdot \operatorname{Pr}[X \leq c] \\
& \leq \mathbb{E}[g(X) \mid X \geq c],
\end{aligned}
$$

where the inequality follows from the fact $\mathbb{E}[g(X) \mid X \leq c] \leq \mathbb{E}[g(X) \mid X \geq c]$. This is true since $g$ is a non-decreasing function, and the conditional distribution 
of $X$, conditioning on $X \geq c$, stochastically dominates the conditional distribution of $X$, conditioning on $X \leq c$ (this is a special case of what we argued in the beginning of the proof of Lem. 1).

To conclude the lemma, let $f(y)$ be the density function of $Y$. Notice that:

$\mathbb{E}[g(X)] \equiv \int_{y} \mathbb{E}[g(X)] f(y) d y \leq \int_{y} \mathbb{E}[g(X) \mid X \geq y] f(y) d y \equiv \mathbb{E}[g(X) \mid X \geq Y]$,

where the equalities follow from the independence of $X$ and $Y$ and the inequality follows from applying (12) pointwise for all $y$.

Proof (of Lemma 3). Let $\mathcal{E}_{i}=\left\{X_{i}=\max _{j}\left\{X_{j}\right\}\right\}$. Then $\mathbb{E}\left[\max _{i}\left\{X_{i}\right\}\right]$ is:

$$
\sum_{i=1}^{n} \mathbb{E}\left[X_{i} \mid \mathcal{E}_{i}, X_{i} \geq t_{i}\right] \operatorname{Pr}\left[\mathcal{E}_{i}, X_{i} \geq t_{i}\right]+\mathbb{E}\left[X_{i} \mid \mathcal{E}_{i}, X_{i} \leq t_{i}\right] \operatorname{Pr}\left[\mathcal{E}_{i}, X_{i} \leq t_{i}\right]
$$

To proceed we need the following claims:

Claim 1: $\operatorname{Pr}\left[\mathcal{E}_{i}, X_{i} \geq t_{i}\right] \geq \frac{p}{1-p} \cdot \operatorname{Pr}\left[\mathcal{E}_{i}, X_{i} \leq t_{i}\right]$.

Proof (of Claim 1).

$$
\begin{aligned}
\operatorname{Pr}\left[\mathcal{E}_{i}, X_{i} \geq t_{i}\right] & =\operatorname{Pr}\left[\mathcal{E}_{i} \mid X_{i} \geq t_{i}\right] \cdot \operatorname{Pr}\left[X_{i} \geq t_{i}\right] \\
& \geq \operatorname{Pr}\left[\mathcal{E}_{i} \mid X_{i} \leq t_{i}\right] \cdot \frac{p}{1-p} \operatorname{Pr}\left[X_{i} \leq t_{i}\right] \\
& =\frac{p}{1-p} \cdot \operatorname{Pr}\left[\mathcal{E}_{i}, X_{i} \leq t_{i}\right],
\end{aligned}
$$

where in the inequality above we used the following facts: First, $\operatorname{Pr}\left[\mathcal{E}_{i} \mid X_{i} \geq t_{i}\right] \geq$ $\operatorname{Pr}\left[\mathcal{E}_{i} \mid X_{i} \leq t_{i}\right]$, which follows from Lem. 1 taking $X=X_{i}, c=t_{i}, g$ the identity function, $Y=\max _{j \neq i}\left\{X_{j}\right\}$ and noticing that the event $\mathcal{E}_{i}$ is the same as the event $X \geq Y$; the second fact we use is that $\operatorname{Pr}\left[X_{i} \geq t_{i}\right] \geq \frac{p}{1-p} \operatorname{Pr}\left[X_{i} \leq t_{i}\right]$, which follows from the fact that $\operatorname{Pr}\left[X_{i} \geq t_{i}\right] \geq p$ and $\operatorname{Pr}\left[X_{i} \leq t_{i}\right] \leq 1-p$.

Claim 2: $\mathbb{E}\left[X_{i} \mid \mathcal{E}_{i}, X_{i} \geq t_{i}\right] \geq \mathbb{E}\left[X_{i} \mid \mathcal{E}_{i}, X_{i} \leq t_{i}\right]$.

Proof (of Claim 2). Just note: $\mathbb{E}\left[X_{i} \mid \mathcal{E}_{i}, X_{i} \geq t_{i}\right] \geq t_{i} \geq \mathbb{E}\left[X_{i} \mid \mathcal{E}_{i}, X_{i} \leq t_{i}\right]$.

From the above claims and the non-negativity of $X_{i}$ it follows that:

$$
\mathbb{E}\left[\max _{i}\left\{X_{i}\right\}\right] \leq \frac{1}{p} \cdot \sum_{i=1}^{n} \mathbb{E}\left[X_{i} \mid \mathcal{E}_{i}, X_{i} \geq t_{i}\right] \operatorname{Pr}\left[\mathcal{E}_{i}, X_{i} \geq t_{i}\right] .
$$

Next we write $\mathbb{E}\left[\max _{i}\left\{X_{i} \cdot \mathbb{I}_{\left(X_{i} \geq t_{i}\right)}\right\}\right]$ as follows:

$$
\begin{aligned}
& \sum_{i=1}^{n} \mathbb{E}\left[X_{i} \mid \mathcal{E}_{i}, X_{i} \geq t_{i}\right] \operatorname{Pr}\left[\mathcal{E}_{i}, X_{i} \geq t_{i}\right]+\mathbb{E}\left[X_{i} \mid \neg \mathcal{E}_{i}, X_{i} \geq t_{i}\right] \operatorname{Pr}\left[\neg \mathcal{E}_{i}, X_{i} \geq t_{i}\right] \\
\geq & \sum_{i=1}^{n} \mathbb{E}\left[X_{i} \mid \mathcal{E}_{i}, X_{i} \geq t_{i}\right] \operatorname{Pr}\left[\mathcal{E}_{i}, X_{i} \geq t_{i}\right]
\end{aligned}
$$

where in the last inequality we used the non-negativity of $X_{i}$. The lemma follows by combining the above lower bound on $\mathbb{E}\left[\max _{i}\left\{X_{i} \cdot \mathbb{I}_{\left(X_{i} \geq t_{i}\right)}\right\}\right]$ with (13). 